\title{
Visualizing intrinsic localized modes with a nonlinear micromechanical array
}

\author{
M. Sato \\ Graduate School of Natural Science and Technology, Kanazawa University \\ Kanazawa, Ishikawa 920-1192, Japan \\ A.J. Sievers \\ Laboratory of Atomic and Solid State Physics, Cornell University \\ Ithaca, NY 14853-2501, USA \\ E-mail: sievers@ccmr.cornell.edu
}

Received November 26, 2007

\begin{abstract}
Micromechanical cantilever arrays provide the opportunity to visualize the nonlinear excitations of a discrete nonlinear system in real time. Both stationary and moving localized nonlinear excitations can be produced either by driving the system at a frequency outside the plane wave spectrum or by driving the system at a frequency within the small amplitude dispersion curve range. To see these modes the tips of the cantilevers are imaged on a 1D CCD camera. The brightness of the image depends on the oscillation amplitude of the cantilever so that a distribution of amplitudes in the array can be recorded as a function of position and time. Both the stationary and traveling excitations have been successfully simulated using a nonlinear lumped element lattice model. The former ILM can appear in any size lattice while the latter requires a low density of modes for the formation of smoothly running excitation.
\end{abstract}

PACS: 05.45.-a Nonlinear dynamics and chaos;

63.20.Pw Localized modes;

63.20.Ry Anharmonic lattice modes;

85.85.+j Micro- and nano-electromechanical systems (MEMS/NEMS) and devices.

Keywords: nonlinear dynamics, intrinsic localized mode, discrete breather, stationary and traveling localized modes, micromechanical cantilever array.

\section{Introduction}

Starting with the works of Lifshitz $[1,2]$ the dynamical properties of defective lattices have been investigated in great detail [3-9]. The general results are that most localized vibrational modes appear outside the harmonic plane wave spectrum and behave as slightly anharmonic oscillators. Even the intricate spectrum of defect modes produced by molecular impurities was unraveled by combining the techniques of persistent IR spectral hole burning with those of FIR spectroscopy [10]. By the 1980's there remained a small group of point defect systems that showed anomalous spectral behavior clearly outside the framework of standard defect model dynamics. One of these systems was KI: $\mathrm{Ag}^{+}$.

The fact that the entire $T=0 \mathrm{~K}$ defect-induced spectrum of $\mathrm{KI}: \mathrm{Ag}^{+}$disappeared as the temperature was increased to $25 \mathrm{~K}$ provided a stringent test of current lattice dynamics theories [11]. The analysis of the temperature dependent results obtained for different vibrational modes provided strong evidence that there were at least two stable elastic configurations associated with an isolated $\mathrm{Ag}^{+}$ion located at a particular $\mathrm{K}^{+}$site in this fcc crystal and that each of these configurations had its own defect-induced vibrational spectrum. By focusing attention on the low-temperature configuration where the $\mathrm{Ag}^{+}$ ion is known to be on-center it was possible to compare theory and experiment in some detail. The theoretical discovery of pocket gap modes and their experimental verification through the host-lattice isotope effect was an important demonstration of the success of the perturbed harmonic approximation in describing this lattice defect system in the low-temperature configuration [12]. However, the theoretical spectrum is still too simple to account for the experimental temperature dependent results since with increasing temperature a variety of experiments in- 
dicated that a different configuration becomes populated in which the $\mathrm{Ag}^{+}$ion is off-center from the normal $\mathrm{K}^{+}$site and tunnels about it. Because of the ion tunneling it is clear that this configuration has a very anharmonic ground state potential. A jump-rotational diffusion model can describe the observed temperature dependent results in terms of two elastic configurations separated from each other by a large energy barrier [13]. The impurity jumps between the configurations with temperature dependent dwell times; however, the physics behind two elastic configurations existing at a single point defect site is still unclear. In 1988 it was proposed that intrinsic localized modes (ILMs) could exist in perfect anharmonic lattices and that the unusual low-temperature spectroscopic properties observed for $\mathrm{KI}: \mathrm{Ag}^{+}$might be a consequence of the trapping of such modes [14]. The proposal was that the defect could act as a vibrational trap for an ILM and it was the capturing or releasing of the ILM at the defect location with temperature that accounted for the unusual spectral behavior observed for this «simple» lattice defect system. A variety of spectroscopic configurations would be a natural consequence of such trapping. Contact was also made with the Lifshitz approach for defect modes. The fact that sometime earlier Kosevich and Kovalev [15] had used a nonlinear Klein-Gordon equation to examine long wavelength localized vibrations in a one-dimensional anharmonic chain was missed by Sievers and Takeno.

Since 1988 ILM experimental and theoretical studies have focused on pure lattices to remove any complexity introduced by extrinsic defects. Published experiments on atomic systems [16-20] present novel spectroscopic signatures assigned to ILMs but without additional evidence supporting localization they do not insure an air tight assignment of localization behavior. The steady state microwave studies of magnetic ILMs in an antiferromagnet, although still spectroscopic in nature, is, perhaps, more convincing since ILM counting [21] and switching [22] have been observed. Reviews of localized excitations for a variety of discrete nonlinear systems have been presented [23-25] and these excitations now appear under a variety of names, such as discrete breather, discrete soliton, or lattice soliton.

The recent development of micro-electrical mechanical systems (MEMS) provides macroscopic nonlinear arrays of both theoretical and practical interest and the direct visualization of the properties of ILMs in these systems is the subject of this paper. Most MEMS applications currently require a linear response but as mechanical beam resonators become smaller, i.e., nano-electromechanical systems (NEMS), their linear dynamic range decreases [26]. Since it is relatively straightforward to drive such small systems into a nonlinear regime [27] another experimental approach makes use of these nonlinear properties in driving and sensing techniques $[28,29]$. The nonlinear vibrational properties of single cantilevers are well understood [30] but the dynamical properties of nonlinearly coupled cantilever arrays with their associated ILMs is under continuing investigation [24].

We have been exploring both by experiment and with simulations the nonlinear excitations of micromechanical cantilever arrays. Their fabrication is fairly straightforward and their oscillation frequencies are in a convenient range: higher than for coupled pendula [31], but lower than other man-made lattices such as Josephson junction arrays $[32,33]$. A 1D CCD camera is used to observe the dynamical behavior of such an array. Under different steady state excitation conditions we have succeeded in producing and observing ILM vibration, in manipulating its position in the lattice and in producing running ILMs. Key components of our findings are described below.

\section{Experimental procedure}

For the mono-element cantilever array modeled as lumped ball and spring elements in Fig. 1, $a$, the zone boundary mode has the highest linear resonant frequency and is characterized by a $\pi$ phase change between vibrating elements. Because our experimental driving arrangement relies on uniform excitation, such a mode is hard to excite. To overcome this coupling problem di-element cantilever arrays have been constructed, composed of long and short cantilevers, as shown in Fig. 1,b. This cantilever array is made from a thin silicon nitride film ( $\sim 300 \mathrm{~nm}$ thick) resting on a silicon substrate. Each cantilever has a transverse vibrational mode and the coupling between cantilevers is provided by thin nitride overhang region. Resonance frequencies are from 60 to $150 \mathrm{kHz}$, and the $Q$ factor is $\sim 10.000$ in vacuum. The number of cantilevers is on the order of 150 and their lengths and pitch is about $50 \mu \mathrm{m}$. Both the cantilever itself and the interconnecting overhang have «hard» or "positive» nonlinearity, i.e., the restoring force becomes larger with increasing amplitude so the resonant frequency increases with increasing amplitude.

The sample is attached to a piezo-electric transducer (PZT) situated in a vacuum chamber to eliminate air damping. For the resonance experiments the array is shaken up and down uniformly. The vibration of each cantilever is recorded by a $1 \mathrm{D}$ CCD camera as shown in Fig. 1,c. A laser beam is line focused along the cantilever tips, and the reflected beam is imaged on the camera using a lens (not shown). As the amplitude of a cantilever increases the overlap of the reflected ray with detector element decreases hence the cantilever image darkens. The array and measuring method are described in more detail in Ref. 24.

The first application of our imaging technique was to measure the linear vibrational dispersion curve of the di-element cantilever array. This experimental informa- 


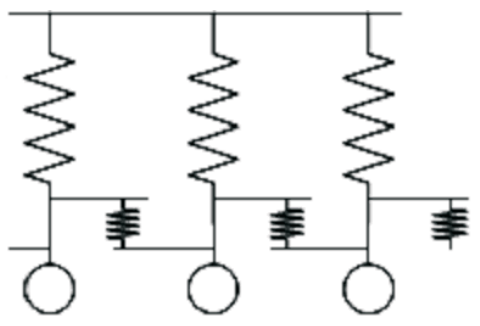

$b$

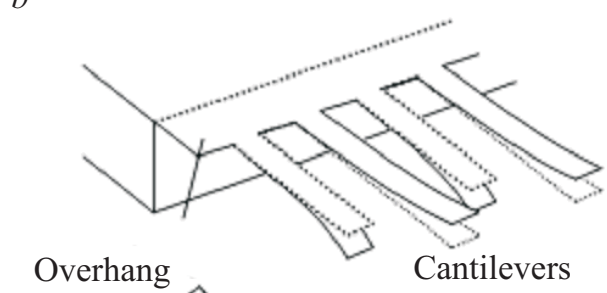

$c$

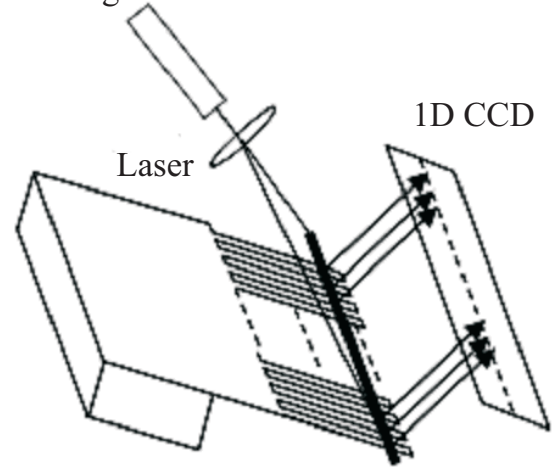

Fig. 1. (a) Coupled ball and spring model. Nonlinear oscillator springs and/or coupling springs are used. (b) The cantilever array is made from a $300 \mathrm{~nm}$ silicon nitride layer. Each cantilever behaves as a nonlinear oscillator coupled together via overhang region. The length and pitch of the cantilevers are about $50 \mu \mathrm{m}$. (c) The observational technique of the cantilever dynamics consists of a line shaped laser beam focused on and reflected from the cantilever tips. This reflected beam is imaged on a $1 D$ CCD camera. When a cantilever acquires a large vibrational amplitude its image darkens.

tion, crucial for the simulations, can be obtained by exciting each linear mode and measuring its wavelength. Since the boundaries at both ends are fixed, the mode shapes are standing waves; one half of the available modes can be excited by the uniform driver. The wavelength of the standing wave can be determined directly from the image.

Several images of standing waves at a low PZT excitation level for a 152 cantilever array were acquired, then Fourier transformed, and the results plotted as a function of the driver frequency. The frequency results as a function of wave number are shown in Fig. 2. By operating the laser in a pulse mode coupled to the driver frequency, the camera integrates images at a fixed phase of oscillation. The fact that the darkness produced by a cantilever is slightly different for the up and down position, enhances the final FFT results so that the modes appear as dark

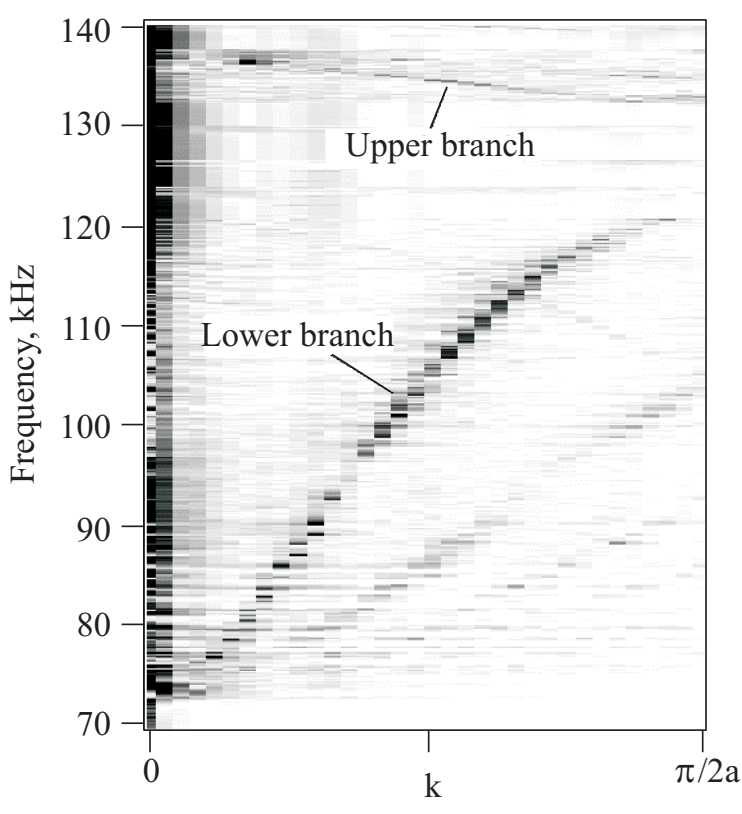

Fig. 2. Experimentally measured dispersion curve of the dielement array. This figure is made from the FFT of images taken as a function of the driver frequency. The upper and lower branches are seen as curves of dark spots. The other curves are the ghosts of the two branches due to nonlinearity in the darkness-response as a function of cantilever displacement.

spots. From this figure, we see that the lower branch ranges from 73 to $120 \mathrm{kHz}$, while the narrower upper branch is from 132 to $137 \mathrm{kHz}$. This asymmetry in bandwidth is due to the long-range interaction between cantilevers produced by the overhang. The small bandwidth of the upper branch helps with the creation of spatially narrow ILMs.

Calculated acoustic and optic-like dispersion curves for the di-element array are shown in Fig. 3. The two bands stem from the high and low resonant frequencies of the two cantilevers. The highest frequency mode located at $k=0$ (uniform) can be excited by the uniform driver, because of the imbalance in mass for this mode shape. The ILM can appear above the upper branch (horizontal thick line in Fig. 3). Since the localized mode is a wave packet, it has a spread in values along the $k$ axis in this dispersion curve picture, the more localized the excitation the larger the spread in $k$ space. The localized shape of the ILM and its relation to the linear dispersion curve has been confirmed by simulations.

The large bandwidth of the lower branch implies a larger spacing between adjacent resonant frequencies, since there are only $152 / 2=76$ modes for each branch. Although the two modes that can be most strongly excited are the highest and lowest frequencies at $k=0$, many of the modes couple to the uniform driving excitation because of the fixed boundary condition. Thus we can excite 


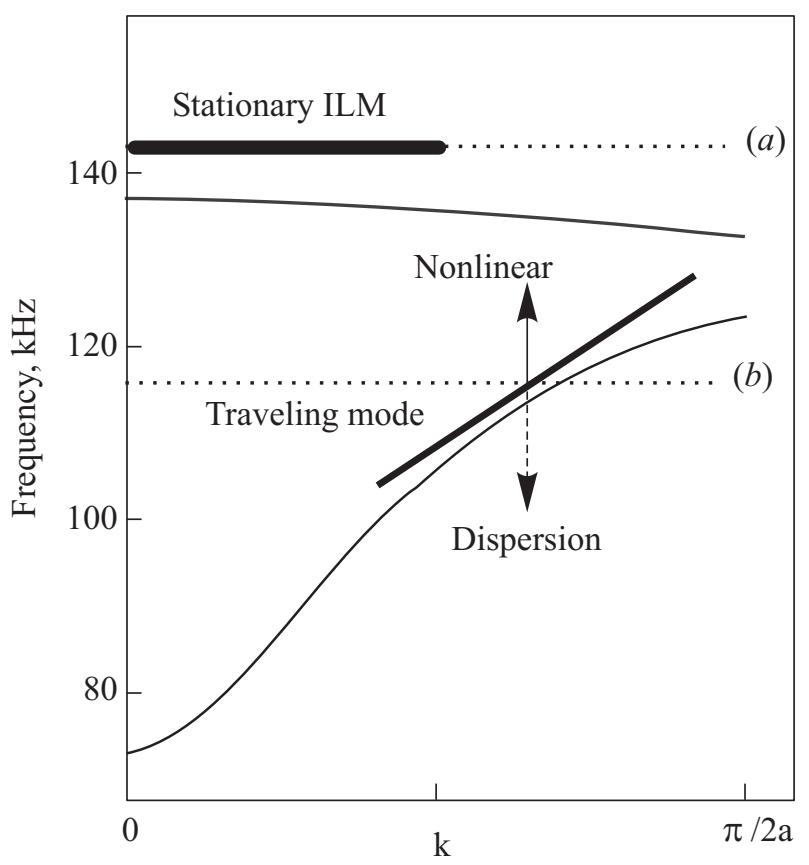

Fig. 3. Dispersion map of the di-element cantilever array. Thin lines identify the linear dispersion curves. The upper branch has a smaller bandwidth because of the effective long-range interaction between cantilevers. The nonlinearity of the array is positive, i.e., the resonance frequencies increase with increasing amplitude. Horizontal dotted curves indicate two different driving frequencies for possible nonlinear excitation $(a)$ where stationary ILMs can appear above the top of the upper branch and $(b)$ where traveling ILMs can be generated. These locked ILMs occur where the dispersion balances the nonlinearity. The straight slanting line identifies the traveling ILM wave packet.

a single mode throughout the lower branch by simply adjusting the driver frequency. The dotted horizontal lines, labeled $(a)$ and $(b)$ in Fig. 3, indicate typical driver frequency location.

\section{Experimental result on intrinsic localized modes}

We have confirmed by experiment that the instability associated with a strongly excited uniform mode causes it to break up into ILMs. This modulation instability $[34,35]$ is seeded by noise, and grows catastrophically replacing the uniform mode with ILMs. Even when the same experimental conditions are maintained, the localization results are different in each run. This result is consistent with the criteria that ILMs do not require impurities and can be located anywhere in the lattice.

A prominent feature of the ILM is its stability at a lattice site. This is related to the energy dependent position in a unit cell [36]. The ILM in this di-element array has the lowest energy state when it is centered at the short cantilever site and hence, it is stable at this site. In at-

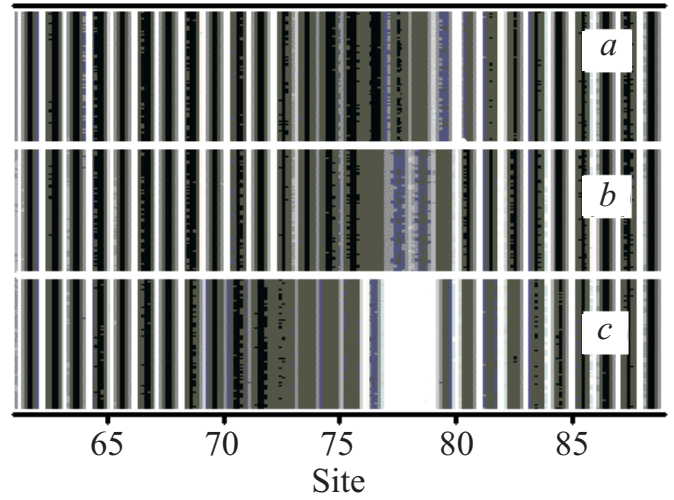

Fig. 4. Optical manipulation of an ILM. The dark patch in frame $a$ identifies a stationary ILM centered at cantilever site 76 . Frame $b$ introduces a low power laser beam focused at site 78 which does not perturb the ILM. Frame $c$ shows that when the power of the laser beam is increased, the ILM is repelled by the laser induced impurity mode and moves to site 72 .

tempting to move to the next short cantilever site, the ILM has to pass over the long cantilever site which requires a higher energy. The energy difference between the two states increases with increasing amplitude. The very stable, stationary ILM seen in Fig. 4, $a$. The dark region near the center of the frame is due to large amplitude cantilevers associated with the ILM. It is obtained by first increasing the driver frequency with respect to the top of the band (up to $1.1 \%$ in a few $\mathrm{ms}$ ), then maintaining a fixed frequency. Increasing the frequency with time from the highest frequency linear mode is a very effective way to obtain a modulational instability. After a number of ILMs are formed, a few remain locked to the driver and grow in strength with time. Such a large amplitude ILM becomes strongly pinned at a lattice site and is very stable. Figure $4, b$ shows that the presence of a weak cantilever heating beam does not modify the ILM because of the locking of the ILM to the driver [37,38]. The vertical white lines are images of cantilevers at rest.

Our success in imaging ILMs leads directly to the possibility of manipulation. The key idea here is to make use of an ILM-impurity interaction. Such an impurity mode can be produced by local heating of a few cantilevers with a focused IR laser beam. Heated cantilevers have a lower resonant frequency due to the temperature dependence of Young's modulus. We have found that an impurity mode produced below the optic-like band repels a nearby ILM, as shown in Fig. 4,c. The IR laser spot is the large white region shown. We have demonstrated that ILMs can be moved in a stepwise manner across the lattice by successive application of this mechanism. In addition, an attractive interaction between a nearby impurity mode and an ILM for an array with negative nonlinearity has also been confirmed [39]. 

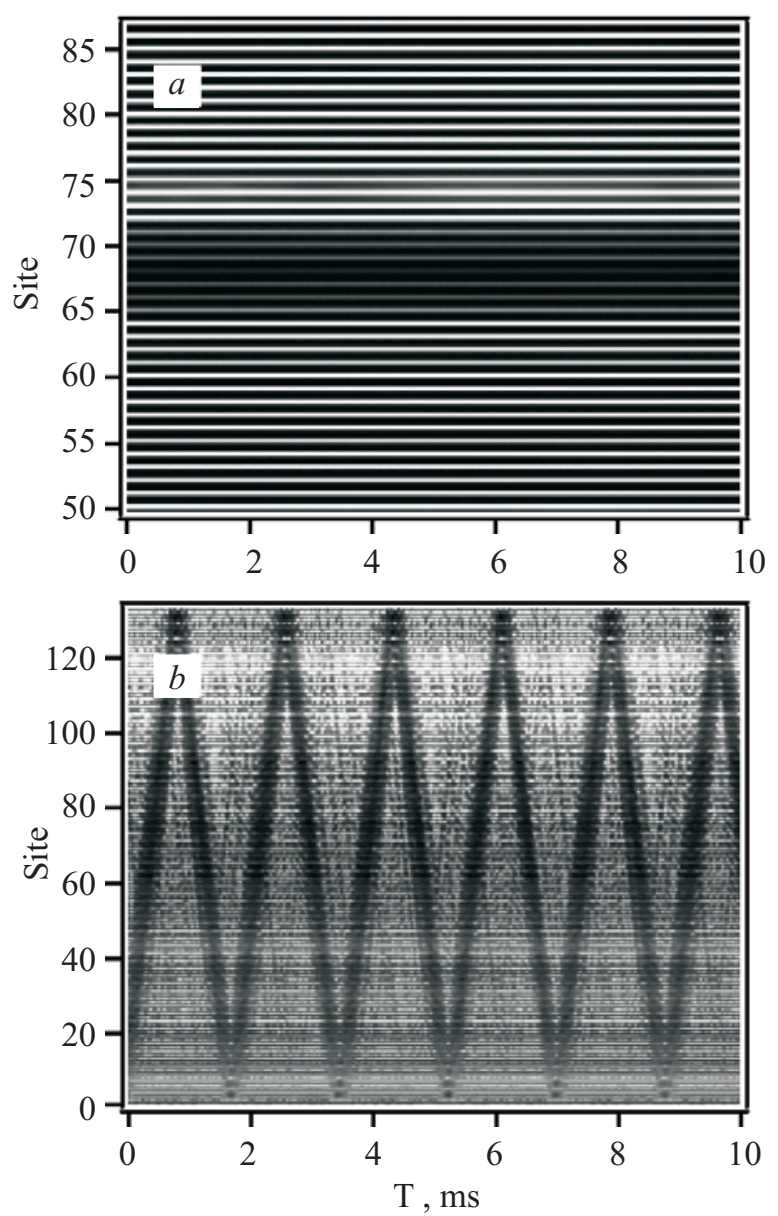

Fig. 5. Experimental observation via site amplitude versus time of two kinds of nonlinear localized excitations for a cantilever array. (a) Stationary ILM. White horizontal lines are images of stationary cantilevers. The ILM is recorded as a dark thick region in the middle of the figure, spread over about seven cantilever sites. It is created via a modulational instability by increasing the driver frequency from the top of the band within a few $\mathrm{ms}$, and then fixing the driver frequency. (b) Traveling ILM. It can be generated in a small system by carefully increasing the driver frequency from a mid resonant frequency toward its neighboring normal mode frequency in the band. Once generated, the localized excitation is very stable. Driving frequency: $137.8(a)$ and $115.41(b) \mathrm{kHz}$.

Figure 5, $a$ shows a stationary ILM for the excitation condition $(a)$ identified in Fig. 3. Now the white line images of cantilevers at rest are horizontal. This picture is to be contrasted with the results for the excitation condition (b) in Fig. 3 that are presented in Fig. 5,b. The dark ILM region traveling and reflecting at the boundaries results in a zigzag pattern in a site versus time plot. Although the lattice has 152 cantilevers, there is a fabrication fault-induced boundary around site 130 . The final state shown in the figure is obtained by carefully tuning the driving frequency. First, it resonantly excites a linear mode. The mode pattern of this resonance is a standing wave because of the boundary condition. Increasing the driver frequency in small steps, causes the amplitude of the standing wave to grow. Similar to the modulation instability observed in case $(a)$ in Fig. 3, the standing wave pattern breaks up into several traveling modes. The initial result appears chaotic. By carefully increasing the driver frequency, often a single traveling mode results. Since the narrow dark region where the excitation level is largest moves through the lattice, it is called a traveling ILM.

Figure 6 illustrates how different excitation patterns may occur depending on the exact initial conditions. First the generation procedure described above produces two colliding ILMs shown panel $(a)$. By increasing the driving frequency by $\sim 1 \%$, see panel $(b)$, the pattern becomes intermittent. A further increase by $0.2 \%$ produces the stable single traveling ILM pattern in panel $(c)$, and finally with another $0.2 \%$ increase, the driver frequency ap-
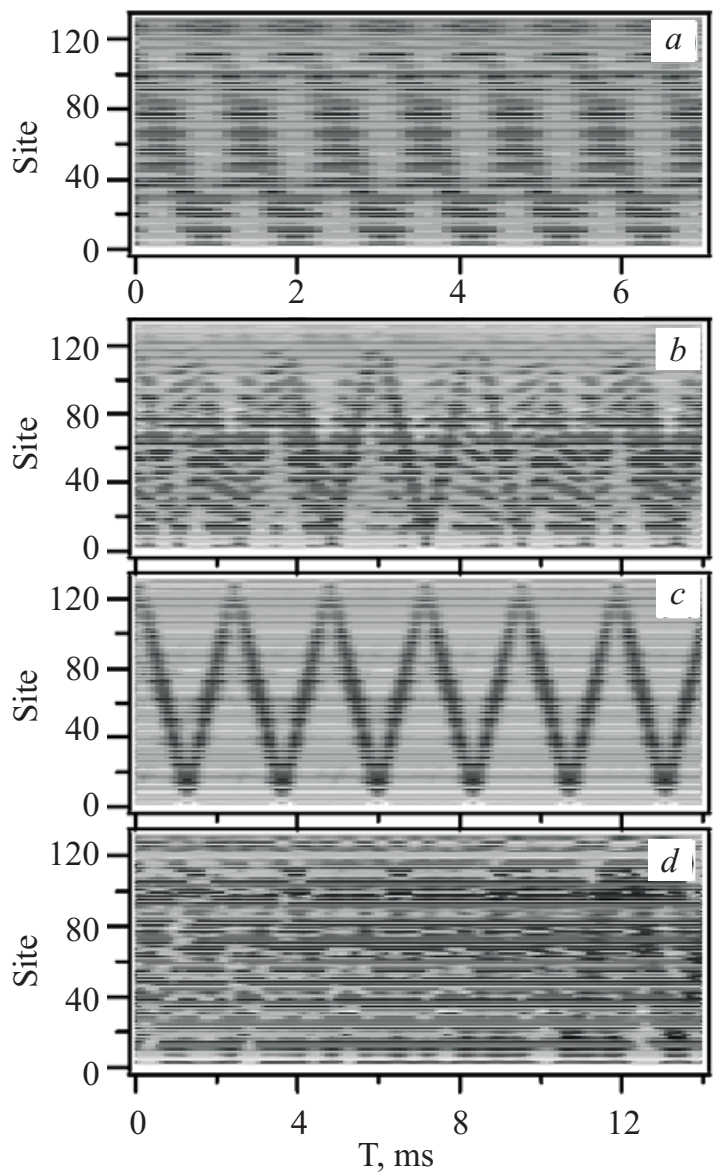

Fig. 6. Several localized excitation patterns for driving frequency locations between two neighboring plane wave values. (a) Two locked traveling modes collide with each other repeatedly, (b) an intermittent ILM pattern, (c) a clean, single locked traveling mode and $(d)$ unstable pattern showing the beating of an ILM with a plane wave mode. Driving frequencies are $116.51(a), 117.39(b), 117.64(c)$, and $117.88(d) \mathrm{kHz}$. The horizontal stripe structure is due to a Moiré effect. 
proaches that of the next plane wave mode of the same symmetry, panel $(d)$, and the pattern again becomes unstable. This series of images was obtained by incrementing the driver frequency at $10 \mathrm{~Hz}$ step. Because of low resolution to reduce the data file, Fig. 6,c looks slightly different from Fig. 5,b. These data were originally taken to check for very long time stability.

We have observed that smoothly running ILMs can only be produced when the monochromatic driver can be tuned within the frequency gap between two modes of the same symmetry suggesting that mobile ILMs are a property of a system with a small number of modes. To check this and other finding we have carried out numerical simulations for arrays of different sizes using the lumped element ball and nonlinear spring model.

\section{Numerical simulations}

To simulate the nonlinear array dynamics, the coupled equations of motion for the nonlinear lumped element model is used, namely,

$$
\begin{aligned}
& m_{i} \frac{d^{2} x_{i}}{d t^{2}}+\frac{m_{i}}{\tau} \frac{d x_{i}}{d t}+k_{20 i} x_{i}+k_{40 i} x_{i}^{3}+ \\
& +\sum_{j} k_{2 I}^{(j)}\left(2 x_{i}-x_{i+j}-x_{i-j}\right)+ \\
& +k_{4 I}\left[\left(x_{i}-x_{i+1}\right)^{3}+\left(x_{i}-x_{i-1}\right)^{3}\right]=m_{i} \alpha \cos (\Omega t),
\end{aligned}
$$

where $m_{i}$ is the mass, $k_{20 i}$ and $k_{40 i}$ are the coefficients of the onsite harmonic and hard quartic potential terms while $k_{2 I}^{(j)}$ and $k_{4 I}$ identify the corresponding intersite terms. More details about the di-element model are given in Ref. 24. The number of lattice sites is 100 with fixed boundary conditions. Model parameters are listed in the second column of Table II in Ref. 24. The equations are integrated forward in time using the fourth order Runge-Kutta routine. At each time step of integration a small amplitude noise field is introduced in order to simulate the effects of random vibrational noise. Figure 7,a shows simulation results obtained with this model. When comparing the experiment results in Fig. 5, $b$ with the simulation in Fig. 7, $a$ a similar time dependence is found. The only difference is that the two ILM speeds are not quite the same. This difference occurs because the slope of the model dispersion curve at the driver frequency is somewhat larger than that observed in experiment. The difference comes about because the driver frequency is $10.6 \mathrm{kHz}$ lower than the top of the lower branch in the simulation, while it is $5.8 \mathrm{kHz}$ from the top for the experiment. A carefully tuning of the driver frequency in the simulation, with sufficient resolution compared to the frequency separation between neighboring plane wave modes, is required to generate a traveling ILM. This ini-
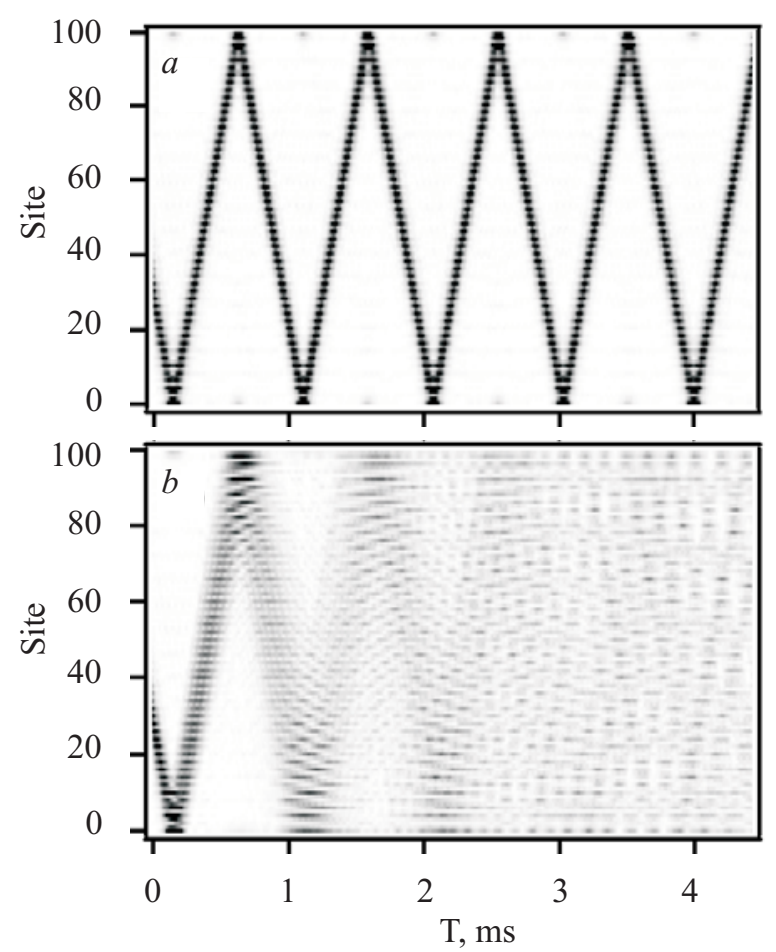

Fig. 7. Simulation results based on a lumped element model. (a) Equations include the nonlinear terms described in Ref. 19. The observed running ILM pattern is quite similar to that found in experiment. (b) Nonlinear terms removed from the equations. Now the localized excitation established at $t=0$ is unstable and breaks up into many plane waves. Same initial conditions for both cases and the driver frequency is fixed at $112.8 \mathrm{kHz}$.

tial condition agrees with that previously used in the experiments.

Additional simulations show that if the array has an insufficient number of elements such smoothly running excitations are suppressed [40].

Figure $7, b$ presents a simulation result with the identical starting condition as that shown in Fig. 7,a, but now the nonlinear terms have been removed so the array is harmonic. The initial localized wave at $t=0$ spreads into many waves with different velocities with increasing time. It is clear that the localized mode envelope is coming apart after only one cycle through the lattice and is no longer well defined after three cycles.

\section{Discussion and conclusions}

The spatial localization of the traveling ILM shown in Fig. 5, $b$ is similar to that found for the stationary ILM, in Fig. 5, $a$. The simulations show that both types of modes can be described by an envelope modulating a carrier wave, but in the latter case the $\omega-k$ display appears as a line tangent to the dispersion curve at the driver fre- 
quency position. Such a situation is represented by the feature near the dotted line $(b)$ in Fig. 3. The speed of the traveling mode can be estimated from experiment, and good agreement is found with the corresponding tangent line. It is to be expected that all such running ILMs can be represented in an $\omega-k$ picture as tangents to the linear dispersion curve, because the dispersion curve identifies the positions of available plane wave modes. The existence of such a traveling ILM depends on a balance between the nonlinear and dispersive effects. Here, the negative curvature of the dispersion balances the positive nonlinearity as represented by the two arrows in Fig. 3. This balance is reminiscent of the soliton stability condition in a continuous nonlinear medium; but, unlike that case, where two solitons can pass though each other with only a phase change, for the lattice problem, in the absence of a driver, energy is transferred between two ILMs during their collision.

These experiments demonstrate that micromechanical cantilever arrays provide a readily accessible technique for exploring the properties of localized excitations in a nonlinear lattice. Two kinds of nonlinear localized excitations have been generated: stationary locked ILMs in the region outside of the plane wave spectrum and traveling ILMs, which appear in the plane wave spectrum. Stationary ILMs can occur for any size discrete system but because locked, smoothly running acoustic ILMs require the driver frequency to occur in the frequency gap between neighboring plane wave modes, practically, they are a property of a small discrete system. These traveling ILMs show some similarities and some differences with bright and dark solitons [41]. The good agreement between experiment and simulations demonstrates that the nonlinear lumped element model captures the important physical signatures that have been observed experimentally.

\section{Acknowledgments}

The authors appreciated the insightful comments of A.M. Kosevich at the 2003 NATO meeting on ILMs at Erice. His suggestion that the ILM spatial oscillations observed in Figs. 8(b,c) of Ref. 23 may be due to Bloch oscillations remains intriguing. We also dedicate this paper to the memory of Shozo Takeno, who pioneered in the ILM field. This work was supported by DOE DE-FG02-04ER46154 and JSPS-Grant-in-Aid for Scientific Research (B) 18340086.

\section{I.M. Lifshitz, J. Phys. (USSR) 7, 249 (1943).}

2. I.M. Lifshitz, Nuovo Cim. Suppl. 3, 716 (1956).

3. A.A. Maradudin, E.W. Montroll, and G.H. Weiss, Theory of Lattice Dynamics in the Harmonic Approximation, F.S.D. Turnbull (ed.), Solid State Physics, 1st ed., Academic Press, New York (1963), Supplement 3.
4. I.M. Lifshitz and A.M. Kosevich, Rep. Prog. Phys. 29, 217 (1966).

5. A.A. Maradudin, in Solid State Physics, F. Seitz and D. Turnbull (eds.), Academic Press, New York (1966), Vol. 19, p. 1.

6. A.J. Sievers, in Localized Excitations in Solids, R.F. Wallis (ed.), Plenum Press, New York (1968), p. 27.

7. R.C. Newman, Adv. Phys. 18, 545 (1969).

8. A.S. Barker and A.J. Sievers, Rev. Mod. Phys. 47, S1 (1975).

9. H. Bilz, D. Strauch, and R.K. Wehner, Vibrational Infrared and Raman Spectra of Non-Metals, L. Genzel (ed.), Handbuch der Physik, Springer-Verlag, Berlin (1984), Vol. XXV, Pt. 2d.

10. W.P. Ambrose and A.J. Sievers, Phys. Rev. B38, 10170 (1988).

11. J.B. Page, D. Schoemaker, H. Fleurent, A.J. Sievers, A. Bouwen, and J.T. McWhirter, Phys. Rev. Lett. 63, 1837 (1989).

12. A. Rosenberg, K.W. Sandusky, B.P. Clayman, J.B. Page, and A.J. Sievers, Phys. Rev. B53, 6076 (1996).

13. R. Lai, C.E. Mungan, and A.J. Sievers, Phys. Rev. Lett. 76, 1864 (1996).

14. A.J. Sievers and S. Takeno, Phys. Rev. Lett. 61, 970 (1988).

15. A.M. Kosevich and A.S. Kovalev, Sov. Phys.-JETP 40, 891 (1974).

16. B.I. Swanson, J.A. Brozik, S.P. Love, G.F. Strouse, A.P. Shreve, A.R. Bishop, W.-Z. Wang, and M.I. Salkola, Phys. Rev. Lett. 82, 3288 (1999).

17. T. Markovich, E. Polturak, J. Bossy, and E. Farhi, Phys. Rev. Lett. 88, 195301 (2002).

18. R.H. Austin, A.H. Xie, L. van der Meer, M. Shinn, and G. Neil, J. Phys.: Condens. Matter 15, S1693 (2003).

19. J. Edler and P. Hamm, Phys. Rev. B69, 214301 (2004).

20. M.E. Manley, M. Yethiraj, H. Sinn, H.M. Volz, J.C. Lashley, W.L. Hults, G.H. Lander, and J.L. Smith, Phys. Rev. Lett. 96, 125501 (2006).

21. M. Sato and A.J. Sievers, Phys. Rev. B71, 214306 (2005).

22. J.P. Wrubel, M. Sato, and A.J. Sievers, Phys. Rev. Lett. 95, 264101 (2005).

23. D.K. Campbell, S. Flach, and Y.S. Kivshar, Physics Today 57, 43 (2004).

24. M. Sato, B.E. Hubbard, and A.J. Sievers, Rev. Mod. Phys. 78, 137 (2006).

25. S. Flach and A. Gorbach, Discrete Breathers-Advances in Theory and Applications, Elsevier Science (2008).

26. I. Kozinsky, H.W.C. Postma, I. Bargatin, and M.L. Roukes, Appl. Phys. Lett. 88, 253101 (2006).

27. M.I. Dykman, C.M. Maloney, V.V. Smelyanskiy, and M. Silverstein, Phys. Rev. E57, 5202 (1998).

28. M. Zalalutdinov, A. Olkhovets, A. Zehnder, B. Ilic, D. Czaplewski, H.G. Craighead, and J.M. Parpia, Appl. Phys. Lett. 78, 3142 (2001).

29. M.V. Requa and K.L. Turner, Appl. Phys. Lett. 88, 263508 (2006).

30. A.N. Cleland, Foundations of Nanomechanics: From Solid-State Theory to Device Applications, Springer, Berlin (2003), p. 312.

31. W. Chen, Phys. Rev. B49, 15063 (1994). 
32. P. Binder, D. Abraimov, A.V. Ustinov, S. Flach, and Y. Zolotaryuk, Phys. Rev. Lett. 84, 745 (2000).

33. E. Truas, J.J. Mazo, and T.P. Orlando, Phys. Rev. Lett. 84, 741 (2000).

34. T. Dauxois and M. Peyrard, Phys. Rev. Lett. 70, 3935 (1993).

35. K.W. Sandusky and J.B. Page, Phys. Rev. B50, 866 (1994).

36. O. Bang and M. Peyrard, Physica D81, 9 (1995).

37. T. Rössler and J.B. Page, Phys. Lett. A204, 418 (1995).
38. M. Sato, B.E. Hubbard, A.J. Sievers, B. Ilic, D.A. Czaplewski, and H.G. Craighead, Phys. Rev. Lett. 90, 044102 (2003).

39. M. Sato, B.E. Hubbard, A.J. Sievers, B. Ilic, and H.G. Craighead, Europhys. Lett. 66, 318 (2004).

40. M. Sato and A.J. Sievers, Phys. Rev. Lett. 98, 214101 (2007).

41. M. Remoissenet, Waves Called Solitons, Third ed., Springer-Verlag, Berlin (1999), p. 76. 